ITACA: ARTÍCULOS DE INVESTIGACIÓN E INNOVACIÓN

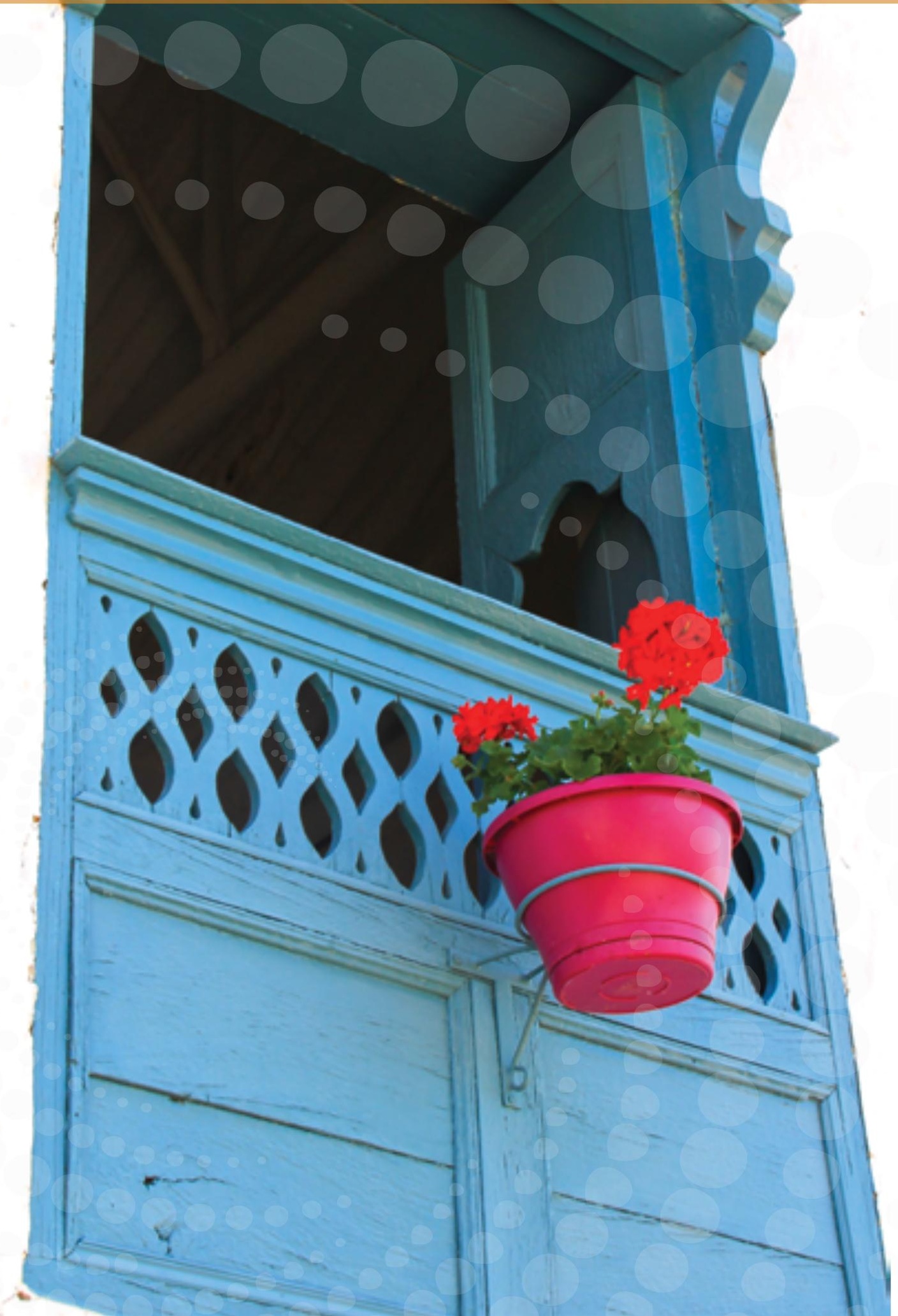





\section{El binomio expectativas-intención en las decisiones académicas en el bachillerato}

\section{The expectations-intention binomial in academic decisions in high school}

\author{
Amparo Romero Valseca \\ Maestría en Tecnología Educativa \\ Correo electrónico: amparo.romero@upaep.edu.mx \\ Universidad Popular Autónoma del Estado de Puebla, México \\ Martha Leticia Gaeta González \\ Doctora en Psicología y Aprendizaje por la Universidad de Zaragoza, España \\ marthaleticia.gaeta@upaep.mx \\ Universidad Popular Autónoma del Estado de Puebla, México
}

Recibido: 17 de mayo de 2018

Aceptado: 20 de junio de 2018

Cómo citar este artículo:

Romero, A., y Gaeta, M.L. (2019). El binomio expectativas-intención en las decisiones académicas en el bachillerato. Espiral, Revista de Docencia e Investigación, 9(1), 11 - 19.

\section{Resumen}

Este trabajo centra la mirada en dos constructos que intervienen en la construcción que los estudiantes de bachillerato hacen de sí mismos, respecto a sus decisiones académicas. El primero, son las expectativas como elemento base, en cuanto a lo que los estudiantes desean realizar en su actividad académica y en las que inciden varios elementos personales y del contexto, que permitirán (o no) concretarla. A partir de ello, creemos necesario considerar, además, a la intencionalidad, para generar acciones concretas hacia el logro de las metas académicas. El objetivo de este estudio es reflexionar sobre la contribución conjunta que las expectativas y la intención tienen en las decisiones académicas de los estudiantes de bachillerato. Con tal propósito, se plantea realizar un recorrido por diferentes aproximaciones teóricas y avances investigativos sobre el tema, a través de una revisión documental. Lo anterior, esperamos nos permita contribuir en nuestro papel como educadores a la comprensión de las decisiones que toman los estudiantes para enfrentar los desafíos y aprovechar mejor las oportunidades de este nivel educativo hacia el éxito académico.

Palabras clave: Aspiraciones, expectativas, intención, decisiones académicas, bachillerato.

\section{Abstract}

This work focuses on two constructs that intervene in the construction that high school students do about themselves, with respect to their academic decisions.
The first is expectations, as a basic element, in terms of what students want to do in their academic activity and in which several personal elements and context affect, which will allow (or not) to achieve it. From this, we believe it is necessary to also consider the intentionality, to generate concrete actions towards the achievement of the academic goals. The objective of this study is to reflect on the joint contribution that expectations and intentions have in the academic decisions of high school students. With this purpose, it is proposed to revise different theoretical approaches and research advances on the subject, through a documentary review. The foregoing hopefully will let us contribute, in our role as educators, to the understanding of the decisions that students take to face challenges and take better advantage of the opportunities of this educational level towards academic success.

Keywords: Aspirations, expectations, intention, academic decisions, high school.

\section{Introducción}

Al igual que muchos países, uno de los retos más grandes que enfrenta México en el ámbito educativo, en términos de cobertura, es ampliar el acceso y reducir el abandono escolar de los alumnos, especialmente en el nivel medio superior. En esta etapa educativa, por sus características, es fundamental la toma de 
decisiones para la vida futura de los estudiantes; concluir los estudios de bachillerato en alguna de las diferentes modalidades ofertadas en el Sistem a Educativo Nacional. Se espera contribuirá a que los jóvenes tengan mejores oportunidades para ingresar al mercado laboral, o bien para continuar su formación académica a nivel superior.

Atender a estas condiciones representa una situación compleja, puesto que existen diversos factores que determinan el éxito o fracaso de los jóvenes en la escuela. En este sentido, Carbajal y Cruz (2015) citan a la directora del gabinete de la OCDE, quien asegura que en México se pierde el $40 \%$ de los jóvenes en la transición de la educación media superior a la superior y a partir de esta etapa se van reduciendo drásticamente los porcentajes de formación académica, hasta llegar a tan solo $12 \%$ de jóvenes entre 20 y 29 años de edad y $4 \%$ de 30 a 39 años que se dedican a estudiar.

A este respecto, Urrutia y Martín del Campo (2015) advierten que cuando en México se habla de abandono escolar y reprobación, durante 2012-2018, se alude predominantemente a la educación media superior (EMS), nivel educativo en el cual se presentan los indicadores más graves. Dentro de este marco se considera oportuno citar a Blanco (2014) quien señala que en México las cifras de acceso al nivel medio superior son relativamente bajas, incluso en comparación con otros países de la región; dado que de una cobertura de cerca de $79.8 \%$ en secundaria desciende a $51.9 \%$ en la EMS. Estas cifras se traducen en que cuatro de cada 10 jóvenes de entre 15 y 17 años de edad no están inscritos en el sistema educativo.

En el ciclo escolar 2000-2001, en la EMS se reportó una matrícula del $56.7 \%$; 12 años después fue de $62.7 \%$. De manera específica, el crecimiento de 2006 al 2013 fue prácticamente nulo. Aunado a estas cifras es necesario considerar en el último período escolar documentado (20122013) una tasa del $15 \%$ de abandono entre y durante ciclos. Asimismo, habría que tener en cuenta el factor regional y el socioeconómico para lograr un panorama más confiable sobre la situación que presenta la EMS en México.

Con el propósito de evidenciar elementos que favorezcan la conclusión de los estudios de los jóvenes, diversos investigadores han centrado su mirada en el estudiante. Los resultados obtenidos presentan evidencia acerca de la implicación que tienen las expectativas y la intención en su consecución académica. Es por ello que en el presente trabajo se busca exponer, de manera reflexiva, diferentes avances sobre las expectativas y la intención en el involucramiento académico y, asimismo, argumentar sobre su implicación conjunta para el éxito académico de los estudiantes de bachillerato.

\section{La conceptualización de expectativas}

Las expectativas representan las percepciones de los individuos acerca de la posibilidad de tener éxito en alguna actividad o tarea (Schunk, 2012), sobre las cuales forja sus proyectos; es decir, la formulación de ideas acerca de qué tan bien les irá en una tarea por emprender. Cabe señalar que este constructo no es sinónimo de competencia percibida, sino que representa las creencias de logro y el valor que tiene una tarea.

Es importante señalar que algunas investigaciones plantean las expectativas y las aspiraciones de manera indistinta. Sin embargo, Elias y Daza (2017) trazan la diferencia entre estos constructos y señalan que las aspiraciones se establecen a largo plazo y las expectativas a corto plazo. Mencionan, además, que la configuración de expectativas puede iniciarse a partir de la influencia de elementos internos y externos de la persona; donde los elementos internos son influenciados por los factores del contexto. También sugieren que la presión normativa que ejercen los grupos sociales, así como la valoración que otros tienen sobre su persona, representan los factores fundamentales en el proceso de conformación de las expectativas, mismas que 
son percibidas como un factor primario a partir del cual se formula una decisión.

De acuerdo con Pérez y Pesántez (2017), las expectativas se van constituyendo a lo largo de la experiencia cotidiana, a través de la cual el sujeto evalúa un conjunto de condicionantes, entre los que destacan la percepción que se tiene de sí mismo, de su situación y de los elementos con los que cuenta para alcanzar las metas que se ha propuesto. Asimismo, formulan que las expectativas contribuyen, en primera instancia, al cumplimiento de los objetivos trazados a futuro.

En este mismo sentido, Pérez-García (2013) expone las contribuciones de Rotter, Chance y Phares, quienes advierten, desde la teoría del aprendizaje social, que las expectativas se formulan tomando en consideración el tipo de situación (interna/externa); de forma tal que, después del éxito en una situación determinada como capacidad (interna), las expectativas se acrecentarían en mayor grado que tras el éxito en una circunstancia de suerte o azar (externa). Asimismo, retoman las aportaciones de Rotter, Liverant y Crowne, quienes sugieren que tras el fracaso de un suceso externo las expectativas se conservan o incluso pueden acrecentar, mientras que tras el fracaso de una situación interna, las expectativas de éxito pueden reducirse. Estas consideraciones sugieren que el grado de internalidad tiene efectos causales que determinan la formulación de las expectativas de una persona en situaciones similares, lo que se sumaría a la valoración de los resultados positivos, así como a la percepción que el sujeto tiene en cuanto a los recursos que posee para hacer frente a las tareas y situaciones, respectivamente.

Por su parte, González y Valenzuela (2016) señalan que las expectativas y aspiraciones realistas corresponden a un proceso de carácter psicológico y social en el que el sujeto hace una valoración objetiva del sentido de competencia que tiene, a partir del cual es atraído hacia una meta, próxima o lejana. Así, las expectativas son determinantes porque asignarán sentido a un propósito educativo que propiciará, a su vez, el motivo para la acción.

\section{Expectativas académicas en el bachillerato}

A partir de los elementos descritos en los párrafos anteriores, abundaremos sobre las expectativas académicas que los estudiantes construyen en el bachillerato para orientar su futuro escolar. Al respecto, Beal y Crockett (2014) han encontrado que las metas y expectativas de los estudiantes se encuentran vinculadas y auguran el nivel educativo que pueden alcanzar. En este mismo sentido, Elias y Daza (2017), a partir de un estudio longitudinal sobre las decisiones de los estudiantes para continuar sus estudios, señalan que las metas académicas se nutren de las aspiraciones y las expectativas. Sin embargo, tomando como base la teoría de las expectativas en su análisis, señalan que las expectativas están apoyadas en el supuesto de que las personas eligen actuaciones que en su opinión las llevarán a los resultados que creen atractivos. Del mismo modo, González (2015) formula que las personas se sienten motivadas cuando creen poder cumplir con una tarea, sea escolar o laboral y, por tanto, las recompensas por recibir serán proporcionales al esfuerzo realizado.

En este mismo sentido, Sepúlveda y Valdebenito (2014) refieren que las expectativas pueden comprenderse como una compleja disposición que circunscribe procedimientos reflexivos y sentidos de posibilidades acerca del futuro, que ubican el quehacer de los actores con relación a sus probables opciones en el campo educativo. Así, las decisiones de acción de los estudiantes se enmarcan de acuerdo con la proyección de lo que pretenden lograr, con base en los medios de los que se cuenta en el momento.

La percepción subjetiva que los alumnos tienen sobre sus oportunidades educativas 
futuras tiene sus bases, por tanto, en la construcción de las expectativas educativas. Derivado de ello, los estudiantes establecen sus decisiones personales para involucrarse en el estudio. Al respecto, Corica (2012) sugiere que las expectativas académicas se basan en experiencias previas que asumen la función de referencia. A partir de esta mirada se toman como punto de partida los acontecimientos del pasado y se construye el horizonte para lo deseable; aquello que se espera como posible sin que necesariamente se tenga certeza de alcanzarlo totalmente. Es por ello que tienden a ajustarse a medida que la posibilidad de satisfacerlas se acrecienta, tal vez porque la conciencia de los problemas interpuestos se torna más aguda.

\section{El concepto de intención}

La intención hacia una acción determinada se ha considerado fundamental en la actuación de las personas. Al respecto, Millán-Puelles (2015) retoma la teoría de Brentano sobre la intencionalidad, definiéndola como la propiedad de los fenómenos psíquicos (intención) frente a los físicos (acción). Desde este enfoque, la psique siempre es intencional, lo que conlleva establecer un carácter consciente de una manera inmediata, es decir, siempre constituye una actitud hacía algo; en este proceso se toma en primera instancia la conciencia de un objeto que habrá de estar enmarcada por una reflexión consiente sobre la acción/actividad por realizar.

Montero (2007) sugiere que las acciones y los pensamientos se encuentran en un proceso constate de transformación que, mediante un giro reflexivo, guían la actitud con tendencia hacia algo en torno a la conciencia. No obstante, incorpora algunos elementos de la teoría de la intencionalidad de Husserl, quien sitúa a la conciencia como fundamento (caracterizada por su tendencia hacia algo) que opera de manera mediadora entre el mundo y el hombre; esto equivale a la alineación de la intencionalidad con la experiencia concreta, lo cual alude a un camino intencional que lleva implícito un lapso consciente.

Por su parte, Gaitán (2009) refiere la teoría de la intención de Bratman, la cual establece que nuestras intenciones inmediatas se forman a partir de una estructura compleja de propósitos orientados hacia el futuro y de un conjunto de normas vinculado a esas estructuras: normas de consistencia, coherencia y estabilidad. Desde este enfoque, las normas tienen la función de posibilitar la capacidad de lograr el mayor número de objetivos; de forma secuencial, en etapas temporalmente ampliadas $y$, tomando en cuenta las limitaciones cognitivas, así como la aparición de circunstancias para deliberar. De esta forma, cuando una intención se implanta convenientemente en una de estas estructuras concretada a partir de planes, la realización, de acuerdo con esas normas, facilita a la persona un alto grado de organización intrapersonal hacia la consecución de los propios fines. Este proceso permite observar que las intenciones previas y sus posteriores realizaciones involucran una referencia de la otra. A manera de precisar, según esta teoría existe un estado mental distinto a los deseos y creencias que se define como un plan, que a su vez será equivalente a la intención.

Desde una perspectiva actitudinal, López (2012) retoma la propuesta de Ajzen, para quien la intención es la decisión de ejecutar un comportamiento o una acción, este elemento será determinante en la conducta de las personas; el sujeto fijará el momento adecuado y de oportunidad en el que realizará un intento para traducir la intención en acción. Así, el sujeto definirá su capacidad de llevar a cabo acciones intencionales o intenciones de actuar.

En este mismo sentido, Morales y Correa (2015) afirman que la intención es considerada como un indicador de la disposición que tiene el sujeto para efectuar una conducta determinada, y es considerada como el antecedente inmediato de la conducta. 


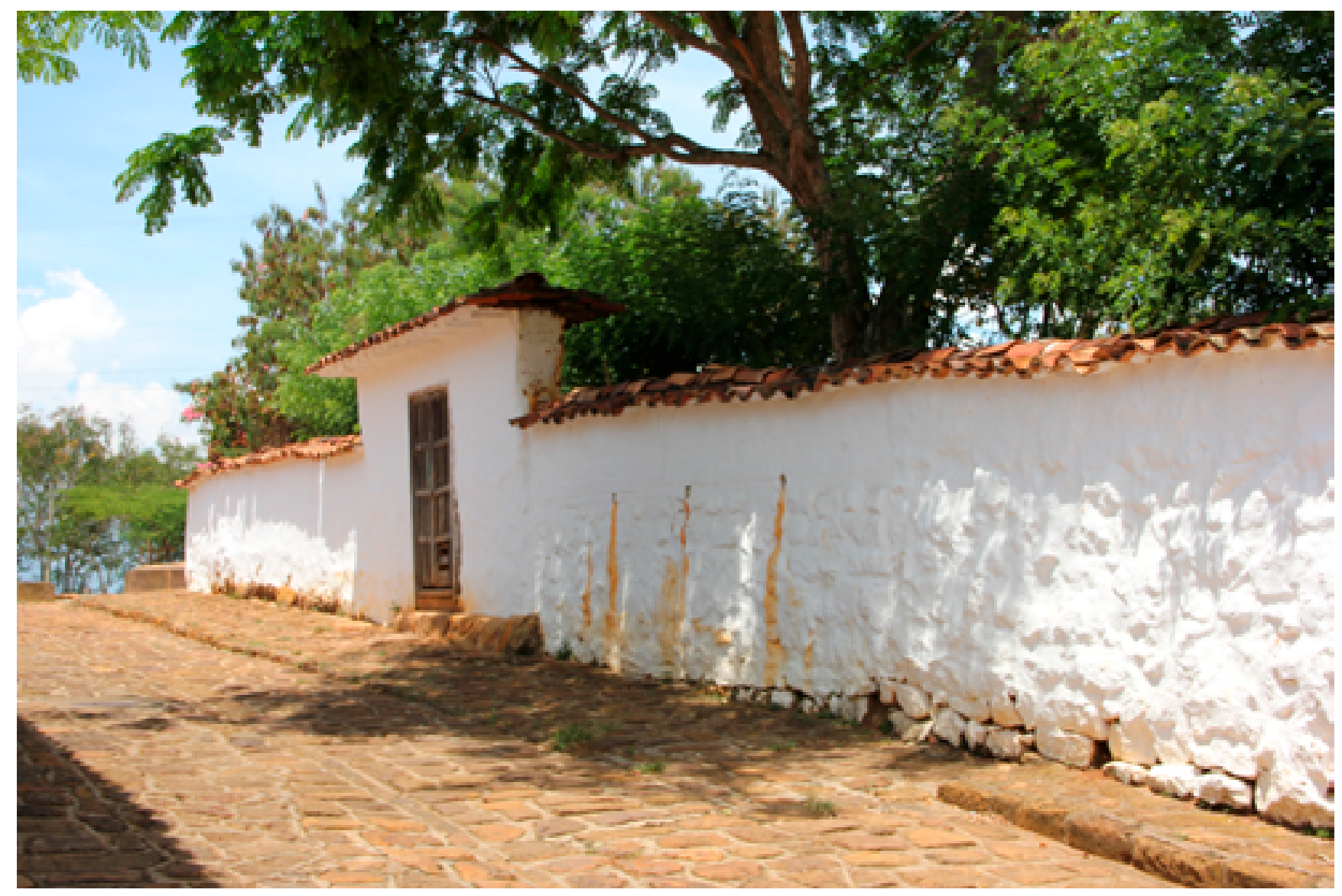

Según las valoraciones anteriores, la intención estará basada en la actitud hacia la conducta, la norma subjetiva y el control conductual percibido, donde cada predictor conductual pesará por su importancia con relación al comportamiento específico y al contexto de interés. En otras palabras, dependiendo del fenómeno, la actitud, la norma, el control o una combinación de estas variables, se puede incidir en mayor medida sobre la intención de manera directa y en consecuencia de alguna forma sobre la conducta.

Tomando como base el modelo tridimensional, mencionado en líneas anteriores, la actitud se construye de tres elementos: el cognitivo, afectivo, y el conativo-conductual.

La actitud hacia la conducta representa la valoración que la persona haga (positiva o negativa) de un comportamiento sobre el cual habrá de decidir y en la que una condición favorable estará asociada a que la intención de llevarla a cabo sea mayor. Así, la actitud estará determinada por las diversas creencias que la persona tenga hacia el objeto (actividad, tarea, persona o institución) y la evaluación que realiza de sí misma. Este valor es el factor afectivo de la actitud, que define a su vez la motivación y la fuerza de la intención de la conducta. Coincidimos con Morales y Correa (2015) en que se pueden tener diferentes creencias, pero estas, por sí solas, no conducen a la acción, por lo que la ponderación de los resultados que se esperan obtener definirán el realizar una acción determinada.

La norma subjetiva se basa en la presión social percibida para tener $o$ no un comportamiento y refleja el efecto de los valores sociales; esto es, está determinada por el componente social del modelo y muestra la influencia de las personas significativas para el 
sujeto, así como la percepción de las creencias, conducta por manifestar y motivación para satisfacer las expectativas que tienen de él o de ella (Fonseca, 2015).

El control conductual refiere a la creencia de la propia capacidad para controlar las dificultades existentes al ejecutar una conducta. Este componente incluye tanto elementos internos como externos de la persona. Dentro de los primeros se hallan las habilidades físicas y psicológicas, y en los segundos el tiempo, la oportunidad, los medios, además de la dependencia de terceros. Se puede decir que el control de la conducta involucra las creencias acerca de la presencia o ausencia de recursos, las oportunidades para tener una conducta y las percepciones de poder controlar los factores que facilitan o dificultan la conducta. Este elemento se instaura con determinante directo tanto para la intención como para la conducta (Huéscar, Rodríguez, Cervelló y Moreno, 2014).

\section{Intención académica en el bachillerato}

En la búsqueda de los objetivos académicos de los estudiantes, se observa un elemento determinante, enmarcado como la intención, que sugiere una actividad futura en cuya esencia se distingue la intervención de la razón. Al respecto, Walton (2016) menciona que la intención tiene sus bases en la razón o conciencia, que se relaciona con la acción práctica y posibilita de forma efectiva la configuración y logro de una meta futura. En este proceso subjetivo, la intención orienta el actuar del sujeto hacia lo mejor posible entre lo previsto y lo alcanzable.

Como plantea Moya (2017), la intención constituye una brújula que guía la acción del sujeto en relación con los fines propuestos, a partir de la representación que le otorga al acto cognoscitivo y que le orienta a ese algo específico. Con base en esta percepción compleja, para efectos de objetivos académicos, el alumno basará en la intencionalidad el impulso que dirija su acción educativa que conlleve la mo- vilización de la capacidad intelectual que posee con miras al logro de las metas académicas propuestas.

Siguiendo con esta línea argumental, una vez establecidos los rasgos que conforman la intención razonada, es preciso el ajuste real con la conducta, para lo que habrá que considerar elementos como: la planificación de una conducta que vislumbre posibles obstáculos, la valoración del individuo sobre los recursos que ha de movilizar, así como las estrategias necesarias sobre el control de la conducta (Bermúdez, 2013). La articulación consciente de todos estos elementos promoverá el logro de las metas académicas de los estudiantes de bachillerato.

En suma, a partir del discernimiento el alumno configurará un plan que encamine sus acciones hacia los fines académicos que se haya propuesto. Por consiguiente, su acción expondrá una intención educativa en sentido pleno. Bajo estas condiciones, la conformación de la vida académica de los estudiantes de bachillerato incluirá como intención la culminación de sus estudios de nivel medio superior. La valoración y el discernimiento del estudiante (actitud, valores y percepción de capacidad para lograrlo) llevarían a las acciones, así como a la movilización de capitales intelectuales de los que dispone. Estos recursos se prevé estarán alineados hacia la consecución de la meta proyectada, a partir de acciones concretas a más corto plazo; asistirá regularmente a sus clases, moverá los recursos de los que disponga para aprobar las asignaturas como el cumplimiento de tareas, exámenes, proyectos, etc., para finalmente concluir exitosamente sus estudios.

\section{El binomio expectativas-intención en las decisiones académicas de los estudiantes de bachillerato}

Con la revisión de las contribuciones descritas en párrafos anteriores, nos permitimos exponer un acercamiento a la configuración del binomio expectativas-intención en las decisio- 
nes académicas de los estudiantes de bachillerato. Como hemos señalado, las expectativas se consideran como procesos reflexivos y juicios de posibilidades acerca del futuro académico de los estudiantes, lo cual implica la movilización de recursos para llevar a cabo acciones a corto plazo. Por su parte, la intención se asume como la valoración que el estudiante hace respecto a la consecución de los estudios y las consecuencias de ese actuar. De esta forma, al vincularse estas, se va configurando una acción conductual determinada mediante un plan razonado, consciente y reflexivo.

Esta condición se puede ejemplificar con el siguiente escenario académico futuro para un jóven de bachillerato. Si se toma como base que el estudiante tiene la expectativa de acceder a estudios universitarios tendrá la intención de terminar sus estudios de manera satisfactoria, por lo que, a partir de una actitud más po- sitiva hacia el involucramiento académico, hará una valoración de su capacidad y recursos para lograrlo, así como de las consecuencias personales y sociales de su acción.

De esta forma, el reconocimiento de los elementos que permiten la configuración del binomio expectativas-intención, sugiere la formulación de acciones encaminadas a identificar, promover y fortalecer, por una parte, las capacidades académicas del estudiante, mismas que sirven como hilo conductor para la formulación de expectativas académicas futuras, seguidas de un proceso mediante el cual se pueda guiar al estuante a establecer un plan estructurado en el que intervengan la razón o conciencia, el control de su actitud, así como la opinión de ciertas personas que tienen importancia para él. Si se cumple con estas condiciones se logrará ayudar a los estudiantes a materializar sus expectativas en intenciones.

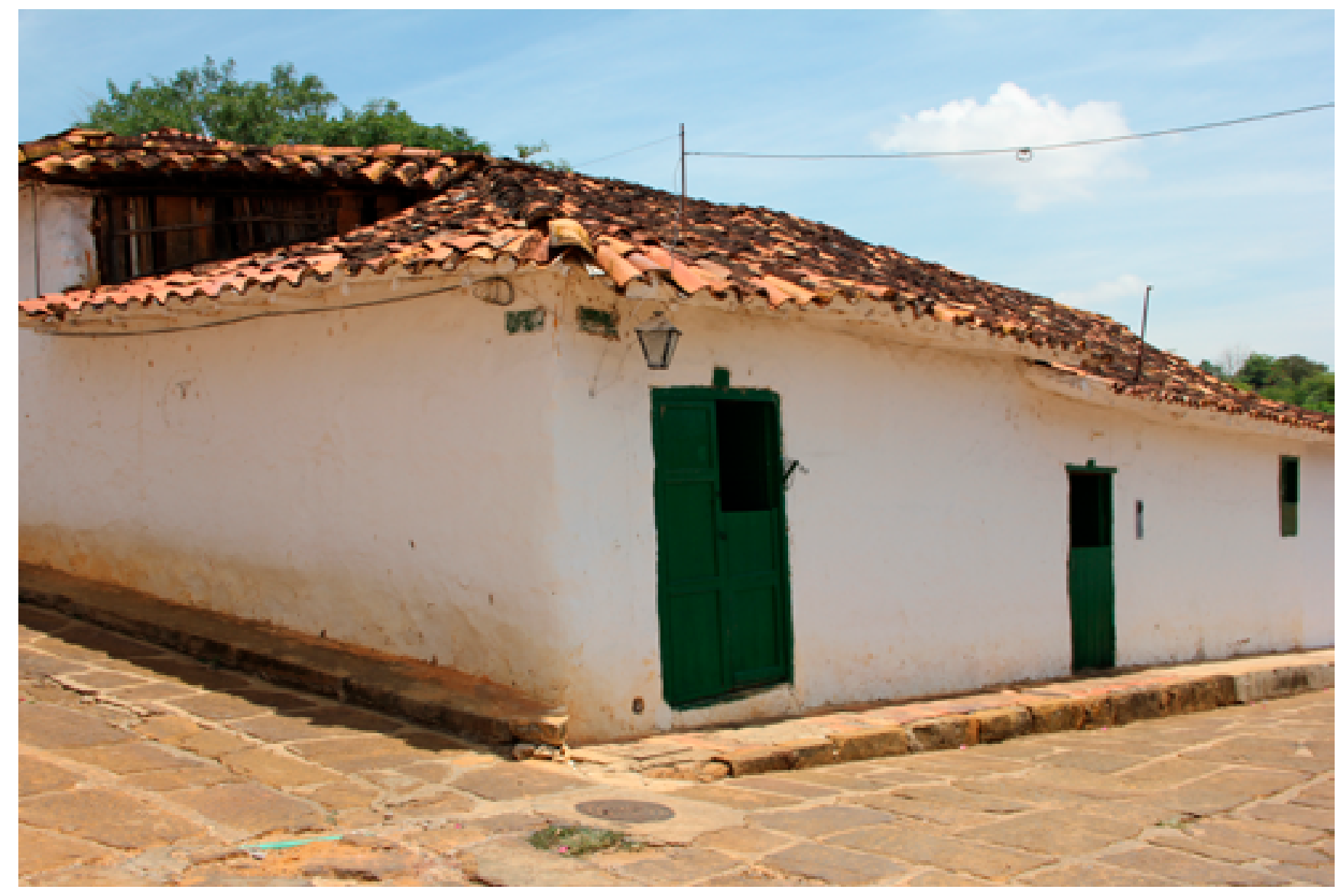




\section{Conclusión}

A partir de las reflexiones vertidas en este trabajo se evidencia la incidencia complementaria entre las expectativas y la intención académica en los planes de los estudiantes, en términos de consecución académica y a la toma de decisiones con base en el razonamiento, la implicación y el compromiso asumidos. Con base en los argumentos aquí expuestos consideramos necesario un mayor estudio al vínculo entre estos dos constructos, considerados como factores determinantes dentro de la configuración del trayecto formativo de los estudiantes, ya que, como hemos señalado, las expectativas no son suficientes para el logro de la acción concreta, si no se cuenta con una intencionalidad, con base en el razonamiento y el compromiso con la consecución de la acción. Se enfatiza por tanto en la necesidad de que en el bachillerato se implementen y fortalezcan acciones académicas que promuevan de una mejor manera la intencionalidad de los estudiantes de continuar su trayecto formativo en el bachillerato y más allá, con base en la generación de mejores expectativas.

\section{Referencias}

Blanco, E. (2014, abril-junio). La desigualdad social en el nivel medio superior de educación de la ciudad de México. Papeles de Población, 20(80). Recuperado de http:// www.redalyc.org/pdf/112/11231067009.pdf

Beal, S., y Crockett, L. (2014). Estudio revela un vínculo entre las esperanzas de los estudiantes con su nivel educativo. Recuperado de https://institutoflash786.org/2014/08/04/ estudio-revela-un-vinculo-entre-las-esperanzas-de-losestudiantes-con-su-nivel-educativo/

Bermúdez, J. (2013). Personalidad, procesos psicosociales y conducta de salud. En J. Bermúdez, A. M. Pérez-García, J. A. Ruiz, P. Sanjuán, y B. Rueda. (Coords.). Psicología de la personalidad. pp. 701-738. Recuperado de https://tuvntana.files.wordpress.com/2015/06/psicologia-de-lapersonalidad-bermudez-perez-y-ruiz.pdf

Carbajal, A., y Cruz, J. (2015). Proyecto de evaluación de los factores de mayor influencia para que los estudiantes de preparatoria abandonen sus estudios, realizado en la preparatoria No. 6, Oaxaca. Recuperado de http:// www.academia.edu/22764861/Universidad_Nacional_ Aut\%C3\%B3noma_de_M\%C3\%A9xico

Corica, A. (2012). Las expectativas sobre el futuro educativo y laboral de jóvenes de la escuela secundaria: entre lo posible y lo deseable. Recuperado de http://www.scielo.cl/ pdf/udecada/v20n36/art04.pdf
Da Fonseca, B. M. (2015). Estudio de la intención emprendedora en Portugal desde la perspectiva de los valores y del género. Recuperado de http://e-spacio.uned.es/fez/ eserv/tesisuned:Psicologia-Bmdfonseca/DA_FONSECA_OLIVEIRA_Belkis_Maria_Tesis.pdf

Elias, M., y Daza, L. (2017). ¿Cómo deciden los jóvenes la transición a la educación postobligatoria? Diferencias entre centros públicos y privados-concertados. Recuperado de https://ojs.uv.es/index.php/RASE/article/ download/9135/9040.

Gaitán, A. (2009, agosto). Planes autonomía y jerarquía. Critica, Revista Hispanoamericana de Filosofía, 41(122), 97-112. Recuperado de https://portal.uc3m.es/portal/ page/portal/grupos_investigacion/hermes/AntonioGaitanTorres/critica_gaitan.pdf

García-Castro, G., y Bartolucci, J. (2007, octubre-diciembre). Aspiraciones educativas y logro académico: Un estudio de caso sobre características y condiciones sociales de los estudiantes de la UAM. Revista Mexicana de Investigación Educativa, 12(35), 1267- 1288 Recuperado de http:// www.redalyc.org/pdf/140/14003507.pdf

González, A., y Valenzuela, J. (2016). Factores motivacionales y construcción de aspiraciones de estudios universitarios: evidencia desde un contexto regional chileno. Recuperado de https://www.researchgate.net/publication/304145830_Factores_motivacionales_y_construccion_de_aspiraciones_de_estudios_universitarios_Evidencia_desde_un_contexto_regional_Chileno

Guzmán, F., García, B., Rodríguez, L., y Alonso, M. (2014). Actitud, norma subjetiva y control conductual como predictores del consumo de drogas en jóvenes de zona marginal del norte de México. Recuperado de http:// www.scielo.org.mx/scielo.php?script=sci_arttext\&pid $=$ S0187-73722014000100003

Huéscar, E., Rodríguez, J., E. Cervelló, E., y Moreno, J. (2014). Teoría de la acción planeada y tasa de ejercicio percibida: un modelo predictivo en estudiantes adolescentes de educación física. Anal. Psicol., 30(2). Murcia. Recuperado de http://scielo.isciii.es/scielo.php?script=sci_artte xt\&pid=S0212-97282014000200037

López, M. (2012). Determinantes de la conducta planeada y el significado del consumo de tabaco y/o alcohol y el consumo de estas sustancias en adolescentes. Recuperado de http://eprints.uanl.mx/3018/1/1080227496.pdf

Millán-Puelles, A. (2015). VII. Obras completas: Léxico filosófico (1984). https://www.rialp.com/libro/ millan-puelles-vii-obras-completas_92846/

Montero, J. (2007, junio). La fenomenología de la conciencia en E. Husserl. Universitas Philosophica, 24(48), 127- 147. Recuperado de http://revistas.javeriana.edu.co/index. php/vniphilosophica/article/viewFile/11271/9185.

Morales, J., y Correa, F. (2015). Modelo de la conducta planeada para predecir la intención de continuar estudiando: Un estudio con adolescentes que viven zona marginal. Recuperado de https://es.scribd.com/document/324851814/ Modelo-de-La-Conducta-Planeada

Moya, P. (2017). Intencionalidad y representación: Comprensión de estos conceptos en la gnoselogía de Tomás de Aquino. Recuperado de http://revistas.ucn.cl/index. php/teologia/article/view/2491/2093

Pérez-García, A. M. (2013). Factores cognitivos y personalidad. En J. Bermúdez, A. M. Pérez-García, J. A. Ruiz, P. San- 
juán y B. Rueda. (Coords.). Psicología de la personalidad. pp. 357-360. Recuperado de https://tuvntana.files.wordpress.com/2015/06/psicologia-de-la-personalidadbermudez-perez-y-ruiz.pdf

Pérez, M. y Pesántez, B. (2017). Impacto migratorio en las aspiraciones y expectativas educativas y de movilidad social de jóvenes Sigseños. Recuperado de http://dx.doi. org/10.17428/rmi.v9i33.242

Portes, A., Aparicio, R., Haller, W., y Vickstrom, E. (2011). Progresar en Madrid: aspiraciones y expectativas de la segunda generación en España. Recuperado de https://dialnet.unirioja.es/servlet/articulo?codigo $=3622422$

Sepúlveda, L., y Valdebenito, M. (2014). ¿Las cosas claras? Aspiraciones de futuro y proyecto educativo laboral de jóvenes estudiantes secundarios. Recuperado de https://scielo.conicyt.cl/scielo.php?script=sci_ abstract\&pid=S0718-07052014000100015\&lng=es\&nr $\mathrm{m}=\mathrm{iso}$
Schunk, D. (2012). Teorías del aprendizaje, una perspectiva educativa. Recuperado https://es.slideshare.net/linoagudelo/teoras-del-aprendizaje-una-perspectiva-educativa-53158955

Ubillos, S., Mayordomo, S., y Páez, D. (2004). Capítulo X. Actitudes: definición y medición componentes de la actitud. Modelo de la acción razonada y acción planificada. Recuperado de http://www.ehu.eus/documents/1463215/1504276/Capitulo+X.pdf.

Urrutia, F., y Martín del Campo, F. (2015). El abandono escolar en el nivel secundaria: un descuido en la agenda educativa actual. Revista Latinoamericana de Estudios Educativos, $X L V(1)$, 63-74. Recuperado de http://www. redalyc.org/pdf/270/27035790003.pdf

Walton, R. (2016). Monadología y teleología en Edmund Husserl. ARETÉ, Revista de Filosofía, 28(1), 145-165. Recuperado de http://www.scielo.org.pe/scielo.php?script=sci arttext\&pid=S1016-913X2016000100007 